\title{
AMENDMENTS
}

\section{Author Correction: Scattering invariant modes of light in complex media}

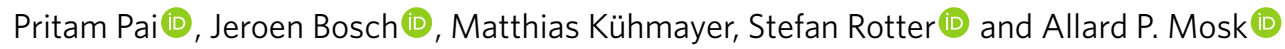

Correction to: Nature Photonics https://doi.org/10.1038/s41566-021-00789-9, published online 8 April 2021.

The Supplementary Information originally published with this Letter was the wrong version, in which the reference list was missing and some parts of sections 4 and 5 had not been updated; this has now been corrected.

Published online: 26 April 2021

https://doi.org/10.1038/s41566-021-00817-8

๑ The Author(s), under exclusive licence to Springer Nature Limited 2021

\section{Author Correction: Hard X-ray transient grating spectroscopy on bismuth germanate}

Jérémy R. Rouxel (1D, Danny Fainozzi, Roman Mankowsky, Benedikt Rösner (D), Gediminas Seniutinas, Riccardo Mincigrucci,

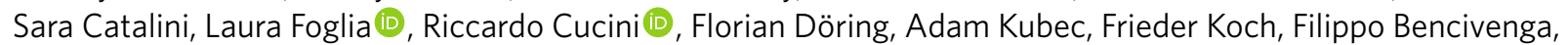
Andre Al Haddad, Alessandro Gessini, Alexei A. Maznev, Claudio Cirelli(D), Simon Gerber (1), Bill Pedrini(D, Giulia F. Mancini, Elia Razzoli, Max Burian, Hiroki Ueda (D), Georgios Pamfilidis, Eugenio Ferrari, Yunpei Deng, Aldo Mozzanica, Philip J. M. Johnson (D), Dmitry Ozerov, Maria Grazia Izzo, Cettina Bottari, Christopher Arrell, Edwin James Divall, Serhane Zerdane, Mathias Sander, Gregor Knopp (D), Paul Beaud (D), Henrik T. Lemke (D),

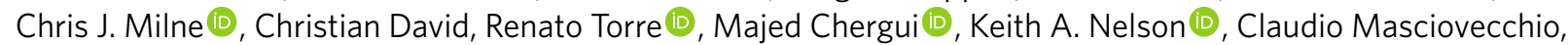
Urs Staub (D), Luc Patthey and Cristian Svetina (D)

Correction to: Nature Photonics https://doi.org/10.1038/s41566-021-00797-9, published online 22 April 2021.

In the version of this Letter originally published, the text 'The spot size at the sample was tuned to $190 \times 150 \mathrm{~mm}^{2}$ ' in the Methods was incorrect; it should have read 'The spot size at the sample was tuned to $190 \times 150 \mu \mathrm{m}^{2}$ '. This has now been corrected in all versions of the Letter.

Published online: 11 May 2021

https://doi.org/10.1038/s41566-021-00826-7

๑ The Author(s), under exclusive licence to Springer Nature Limited 2021

\section{Author Correction: High-sensitivity imaging of time-domain near-infrared light transducer}

Yuyang Gu®, Zhiyong Guo, Wei Yuan, Mengya Kong, Yulai Liu, Yongtao Liu®, Yilin Gao, Wei Feng (1), Fan Wang(D), Jiajia Zhou (D), Dayong Jin (1) and Fuyou Li (i)

Correction to: Nature Photonics https://doi.org/10.1038/s41566-019-0437-z, published online 20 May 2019.

In the version of this Letter originally published, there were some mistakes in the Supplementary Information file. In Supplementary Fig. 11, the labels of the brown and green lines incorrectly read ' $\beta-\mathrm{NaYbF}_{4}, \mathrm{Tm}_{\mathrm{C}} \mathrm{CaF}_{2}$ ' and ' $\beta-\mathrm{NaYbF}_{4}, \mathrm{Er} @ \mathrm{CaF}_{2}$ ', respectively; they should have read ' $\beta-\mathrm{NaYbF}_{4}, \mathrm{Tm} @ \mathrm{NaYF}_{4}$ ' and ' $\beta-\mathrm{NaYbF}_{4}, \mathrm{Er} @ \mathrm{NaYF}_{4}$ '. In Supplementary Fig. 13, the day 7 images were duplicates of the day 0 images; the correct images have now been inserted. In Supplementary Fig. 14, the panel labels a and b were missing; they have now been added. In Supplementary Table 3, in the 'Radiative Lifetime (ms)' column, the average value was incorrectly given as $2.42 \mathrm{~ms}$; it should have been $2.43 \mathrm{~ms}$. The Supplementary Information file has now been replaced with the updated version.

Published online: 9 June 2021

https://doi.org/10.1038/s41566-021-00834-7

(C) The Author(s), under exclusive licence to Springer Nature Limited 2021 